\title{
Iron as a Tracer in Galaxy Clusters and Groups
}

\author{
Alvio Renzini ${ }^{1}$ \\ European Southern Observatory, \\ Karl-Schwarzschild-Strasse 2, D-85748, Garching bei München, Germany, \\ arenzini@eso.org
}

Received __; accepted _

To appear in ApJ, October 10, 1997

${ }^{1}$ On leave from: Dipartimento di Astronomia, Università di Bologna, Italy. 


\begin{abstract}
Available X-ray data are collected and organized concerning the iron and gas content of galaxy clusters and groups, together with the optical luminosity, mass and iron abundance of cluster galaxies. Moving from such a restricted number of cluster parameters several astrophysical inferences are drawn. These include the evidence for rich clusters having evolved without much baryon exchange with their surrondings, and having experienced very similar star formation histories. Groups are much gas-poor compared to clusters, and appear instead to have shed a major fraction of their original cosmic share of baryons, which indicates that galaxy clusters cannot have formed by assembling groups similar to the present day ones. It is argued that this favors low- $\Omega$ universes, in which the growth of rich clusters is virtually complete at high redshifts. It is also argued that elemental abundances in clusters are nearly solar, which is consistent with a similar proportion of supernovae of Type Ia and Type II having enriched both the solar neghborhood as well clusters as a whole. Much of the iron in clusters appears to reside in the intracluster medium rather than inside galaxies, the precise ratio being a function of the Hubble constant. It appears that the baryon to star conversion in clusters has been nearly as efficient as currently adopted for the universe as a whole. Yet the metallicity of the clusters is $\sim 5$ times higher than the global metallicity adopted for the nearby universe. It is concluded that the intergalactic medium should have a metallicity $\sim 1 / 3$ solar if stellar nucleosynthesis has proceeded in stars within field galaxies with the same efficiency as in stars within clusters of galaxies.
\end{abstract}

Subject headings: galaxies: abundances - galaxies: clusters: general - galaxies: formation - galaxies: intergalactic medium 


\section{Introduction}

The X-ray observations of clusters of galaxies have revealed the presence of large amounts of iron and other heavy elements in the intracluster medium (ICM) (Mitchell, Ives, \& Culhane 1975; Serlemitsos et al. 1976; Mushotzky et al. 1996), thus providing direct evidence that gas contaminated by nucleosynthesis processes has been lost by galaxies in the course of their evolution. The access to the ICM elemental abundances has offered the opportunity to investigate several important phenomena, such as the baryon circulation on various scales (from galaxies to clusters), the integral past supernova (SN) activity along with the relative role of the two major SN types, the efficiency of gas to galaxies, stars, and metals conversion at a cluster scale, etc. (e.g., Vigroux 1977; Matteucci \& Vettolani 1988; Arnaud et al. 1992; Renzini et al. 1993, hereafter RCDP; Loewenstein \& Mushotzky 1996). Some of these issues have been extensively discussed in RCDP (see also Renzini 1994) on the basis of empirical evidence coming almost exclusively from Einstein X-ray observations. In more recent years a great deal of relevant new data have become available from the ROSAT and ASCA satellites, and in this paper some of the issues discussed by RCDP are revisited, and some new inferences are drawn.

Clusters are the largest objects on which chemical enrichment can be thoroughly studied, and offer the additional advantage of being perhaps the best example in nature for which the closed box approximation may hold true. Pei \& Fall (1995) have recently modeled the chemical evolution of damped Ly $\alpha$ absorbers, thus predicting the evolution of the global star formation rate with redshift (cosmic time). Global star formation rates all the way to $z \simeq 1$ and beyond have been empirically constructed by Madau et al. (1996) using data from the Canada-France Redshift Survey (Lilly et al. 1996), and found it in remarkable agreement with the predictions of Pei \& Fall. Yet, absorbers represent a minor fraction of the baryonic matter, unlikely to be well approximated by the closed box model, 
and Pei \& Fall allow for both inflow and outflow in their chemical evolution model. Clusters of galaxies are to some extent complementary to Ly $\alpha$ absorbers, in that they contain a large amount of heavy elements, partly in the hot ICM, partly locked into stars, none of which participates in absorbing quasar light. With a bottom-up approach, in this paper the iron (and metal) content of clusters and groups is used as a tracer of baryon circulation, past star formation, and supernova enrichment at the cluster scale, and beyond.

The paper is structured as follows: Section 2 presents a recollection of literature data concerning the iron and gas content of X-ray clusters and groups, that will form the factual basis for the considerations to be developed in the subsequent sections. In Section 3 several inferences are derived from the uniformity of these cluster properties, including the evidence for clusters having experienced little baryon-exchange with their surrondings, having turned gas into galaxies with nearly constant efficiency, and having experienced very similar star

formation histories. Groups instead present a completely different scenario, which suggests that present day clusters cannot have formed by coalescence of groups similar to the present day ones. Section 4 deals with the elemental relative abundances at the cluster scale, while Section 5 deals with the global metallicity of clusters versus that of the present day universe as a whole, and with the global star formation history of the universe. The main conclusions of this paper are finally listed in Section 6.

\section{The Iron Mass to Light Ratio of Clusters and Groups}

Ciotti et al. (1991) introduced the concept of ICM iron mass to light ratio $(\mathrm{Fe} M / L)$ as the ratio $M_{\mathrm{Fe}}^{\mathrm{ICM}} / L_{\mathrm{B}}$ of the total iron mass in the ICM over the total optical luminosity of galaxies in the cluster, and found values in the range $(0.7-1.6) \times 10^{-2} M_{\odot} / L_{\odot}$ for the nearby clusters Virgo, Coma, and Perseus. On a wider database, Arnaud et al. (1992) found $M_{\mathrm{Fe}}^{\mathrm{ICM}} \propto L_{\mathrm{B}}$, and RCDP finally adopted $M_{\mathrm{Fe}}^{\mathrm{ICM}} / L_{\mathrm{B}}=0.01-0.02 M_{\odot} / L_{\odot}$ as typical 
for rich clusters. This value has a moderate dependence on the Hubble constant, being proportional to $h^{-1 / 2}$ (Renzini 1994); for the data presented in this Section $h=1 / 2$ has been adopted. The Fe $M / L$ relates two pieces of fossil information: the integral amount of iron ejected by galaxies in the course of their evolution, and the present luminosity of the old stellar remnant of the population that preasumably produced the observed iron, early in its evolution.

The iron mass in the ICM is obtained as the product $Z_{\mathrm{ICM}}^{\mathrm{Fe}} M_{\mathrm{ICM}}$ of the iron abundance times the mass of the ICM gas. The iron abundance is generally estimated at the center of the cluster, and one assumes the ICM to be chemically homogeneous. This is the case for rich, high temperature clusters, while radial gradients in iron abundance have been reported for cooler clusters from $A S C A$ data (Ohashi et al. 1995). In principle, it is possible to obtain $M_{\mathrm{Fe}}^{\mathrm{ICM}}$ integrating radially $Z_{\mathrm{ICM}}^{\mathrm{Fe}}$ in $\rho d V$. However, $A S C A$ 's PSF does not allow a good reconstruction of the cluster ICM density profile. Such integration could be accomplished by combining $A S C A$ data with those of other X-ray telescopes with better PSF.

Figure 1 shows $M_{\mathrm{Fe}}^{\mathrm{ICM}} / L_{\mathrm{B}}$ as a function of the cluster total luminosity. For $L_{\mathrm{B}} \gtrsim 4 \times 10^{11} L_{\odot}$ the $\mathrm{Fe} M / L$ appears indeed to be constant, with very small scatter around an average of $\sim 0.02 M_{\odot} / L_{\odot}$. However, for $L_{\mathrm{B}} \lesssim 4 \times 10^{11} L_{\odot}-$ i.e., in poor groups rather than rich clusters - the Fe $M / L$ exhibits much smaller values, with no good correlation with the cluster optical luminosity. Figure 2 shows the same $M_{\mathrm{Fe}}^{\mathrm{ICM}} / L_{\mathrm{B}}$ data now plotted as a function of the temperature of the X-ray ICM. It is now apparent that the $\mathrm{Fe} M / L$ is virtually constant all the way to temperatures down to $\sim 1 \mathrm{keV}$, and then drops precipitously by almost three orders of magnitude as the temperature decreases below 1 $\mathrm{keV}$. The separation between groups and clusters is striking in this diagram. It is worth mentioning that groups shown in this figures represent a biased sample in that they are 
selected for being detected in X rays. Typically, spiral rich groups (similar to the Local Group) are not detected in X rays, which may be due to them having an even lower ICM content, lower ICM mass to light ratio, and therefore lower $\mathrm{Fe} M / L$.

The drop of the derived Fe $M / L$ in poor clusters and groups can be traced to a drop in both factors entering in its definition, i.e., in the iron abundance and in the ICM mass to light ratio. Figure 3 shows indeed ICM mass to light ratio $M_{\mathrm{ICM}} / L_{\mathrm{B}}$ as a function of the cluster optical luminosity. While rich clusters have fairly constant ICM mass to light ratio, much lower values with large dispersion are exhibited by poor clusters and groups with $L_{\mathrm{B}} \lesssim 4 \times 10^{11} L_{\odot}$. The ICM mass is typically measured within a radius that scales with the optical radius of the cluster, and therefore it is smaller for groups than for clusters. Figure 4 shows $M_{\mathrm{ICM}} / L_{\mathrm{B}}$ as a function of the ICM temperature. Again, fairly constant values $\left(M_{\mathrm{ICM}} / L_{\mathrm{B}} \simeq 30 M_{\odot} / L_{\odot}\right)$ are exhibited by clusters hotter than $\sim 1 \mathrm{keV}$, while the ICM mass to light ratio drops precipitously below $\sim 1 \mathrm{keV}$.

Figure 5 shows the iron abundance $Z_{\mathrm{ICM}}^{\mathrm{Fe}}$ as a function of the cluster optical luminosity. Again, rich clusters with $L_{\mathrm{B}} \gtrsim 4 \times 10^{11} L_{\odot}$ exhibit a fairly constant iron abundance, at the level of $\sim 0.3$ solar. Poorer clusters instead show a large dispersion, with very low values being reached among poorest clusters. The iron abundance is finally shown as a function of ICM temperature in Figure 6. Clusters hotter than $\sim 2.5 \mathrm{keV}$ have similar abundances, with very small dispersion. Going to cooler clusters $Z_{\mathrm{ICM}}^{\mathrm{Fe}}$ appears first to increase up to near solar abundance for $k T$ slightly in excess of $1 \mathrm{keV}$, and then drops precipitously to almost zero below $1 \mathrm{keV}$, with a fairly strong abundance-temperature correlation (see also Arimoto et al. 1997, hereafter AMIOR). The presence of this correlation shows that just larger statistical errors at low $k T$ cannot account for the apparent large spread of abundances. As extensively discussed by AMIOR, very low iron abundances are also derived from X-ray data for the ISM of ellipticals, which all have temperatures $\lesssim 1 \mathrm{keV}$. Also among ellipticals 
the iron abundance appears to correlate with temperature, the lower the temperature the lower the estimated abundance (e.g., Davis \& White 1996).

It is apparent from all these figures that the $\mathrm{Fe} M / L$, the ICM mass to light ratio, as well as the iron abundance all correlate much stronger with cluster temperature rather than with optical luminosity. Part of the problem may be due to observational errors, as cluster optical luminosities are difficult to determine. However, there may be other effects at work to make so strong the correlation with temperature. Some will be explored in the next sections.

\section{The Baryon Circulation in Clusters and Groups}

\subsection{Rich Clusters}

The constancy of the Fe $M / L$ and of abundance among clusters give support to the notion that galaxy clusters neither lost baryons to, nor acquired baryons from the outside in the course of their evolution. Were clusters loosing gas at some stage (e.g., as a result of the ICM heating by galactic winds or AGNs) then iron would be lost as well, and sizable cluster to cluster differences in the Fe $M / L$ should arise. Seemingly, were clusters to accrete pristine gas $\left(Z^{\mathrm{Fe}} \simeq 0\right)$ in substantial amount then iron would be diluted, and cluster to

cluster differences in $Z_{\mathrm{ICM}}^{\mathrm{Fe}}$ would arise. Of course, one may argue that all clusters had nearly the same amount of baryon exchange with their surrondings, which however seems a rather contrived requirement. This latter empirical result is in qualitative agreement with the theoretical prediction according to which the baryon fraction of clusters cannot change appreciably in the course of their evolution (White et al. 1993; Evrard 1997).

The constancy of the FeM/L, ICM mass to light ratio, and abundance altogether suggest that the conversion of baryons into galaxies, stars and finally metals took place 
with nearly the same efficiency and at the same epoch in all clusters. Indeed, the constancy of the $\mathrm{Fe} M / L$ says that the same amount of iron was ejected by galaxies per unit present stellar population. The most direct interpretation is that there are no appreciable cluster to cluster differences in the global stellar initial mass function (IMF) and star formation history, unless variations in IMF are rather precisely compensated by variations in the distribution of stellar ages (again, a rather contrived requirement). For example, a difference by $\Delta x=0.5$ in the IMF slope would imply a factor $\sim 4$ difference in the $\mathrm{Fe} M / L$ (cf. RCDP). Differences from cluster to cluster in the average age of the stars producing the present optical luminosity of the cluster would also affect the FeM/L. Assuming the cluster luminosity evolution to be dominated by the passive aging of the elliptical galaxy population $\left(L \propto \simeq t^{-(4-x) / 3}\right)$, a factor of two difference in average stellar age implies a similar difference in luminosity, hence in the $\mathrm{Fe} M / L$.

As well known, most of the cluster light $L_{\mathrm{B}}$ is provided by elliptical galaxies and galactic spheroids. Moreover, they represent an even larger fraction of the stellar mass content of clusters since these passively evolving systems have larger $M / L_{\mathrm{B}}$ ratios than star forming galaxies. It is now well established by several independent lines of evidence that the bulk of stars in cluster ellipticals formed at high redshift, i.e., $z \gtrsim 3$ (for a review see e.g., Renzini 1995). This comes from the tightness of the color $-\sigma$ relation of ellipticals in nearby clusters (Bower, Lucey, \& Ellis 1992), the tightness of the distributions of elliptical galaxies about their fundamental plane at low and high redshift (Renzini \& Ciotti 1993; van Dokkum \& Franx 1996; Pahre, Djorgovski, \& de Carvalho 1997), the tightness of the $\mathrm{Mg}_{2}-\sigma$ relation, again at low as well as high redshift (Bender, Ziegler, \& Bruzual 1996), the existence of red (elliptical) galaxies at high redshift (Hamilton 1985; Aragon-Salamanca et al. 1993; Dickinson 1995), and the tightness of the color-magnitude relation of cluster ellipticals at high redshift (Ellis et al. 1996). The possibility has been advocated of fine tuning between age and metallicity effects conspiring to preserve the tightness of these 
relations at low- $z$ while allowing for a large age spread (Worthey, Trager, \& Faber 1995). This appears to be ruled out by the observation that the tightness is preserved even for clusters at large lookback times, while it would obviously be destroyed if a large age spread was present among ellipticals in low- $z$ clusters (Kodama \& Arimoto, 1997).

It is most straigtforward to identify in the spheroidal populations the producers of the bulk of the ICM iron in clusters, since most of rich cluster stellar luminosity comes from ellipticals, S0s, and the bulges of early-type spirals (which are also dominated by very old stellar populations, cf. Ortolani et al. 1995; Jablonka \& Alloin 1995). If so, then the bulk of iron had to be produced by stars and ejected from galaxies at very early times, say at $z \gtrsim 2-3$, even if a major fraction of iron could have been produced by late SNIa exploders (RCDP). This - coupled with a non-evolving baryon fraction of clusters (White et al. 1993) - implies an ICM iron abundance independent of redshift, all the way to very high redshifts. Some evidence in this direction is indeed emerging, though for the moment limited to moderate redshifts (cf. Figure 6).

Although it appears most natural to attribute to ellipticals and spheroids the prime role as iron (metal) producers in clusters, yet other alternatives have been entertained and are worth considering. Giant elliptical galaxies and large spheroids have a rather deep potential well, hence ejecting large amounts of metals requires a great deal of energy. Dwarfs instead de-gas much more easily than giants, having much shallower potential wells, and therefore dwarfs (e.g., dSph galaxies) are likely to have ejected more iron per unit present light than giants. This is indeed a common feature of galactic wind models for the formation of ellipticals and spheroids (e.g., Larson 1974; Arimoto \& Yoshii 1987; Matteucci 1992). Clearly, the problem is whether there were enough dwarfs to make an appreciable fraction of the ICM iron. It has actually been argued that most ICM itself might have been ejected from dwarfs, given that the dwarfest among dwarf spheroidals have a very low baryonic 
fraction, hence had to eject most of their baryons if they were to start with the cosmic share (Trentham 1994). However, both the faint end of the galaxy luminosity function, and the relative amounts of baryons and metals ejected by dwarfs are sufficiently uncertain to make very hard either to prove or disprove this scenario. Most baryons are now in the ICM rather than within galaxies (i.e., galaxies contain $\sim 20 \%$ of the baryons, e.g., David et al. 1990; White et al. 1993). Hence, the question is whether the baryons were separated from their (small scale) dark matter complement by local starbursts, or by mutual stripping in the process of hierarchical clustering (Nath \& Chiba 1995), when small scale density peaks dissolved inside the collapsing cluster. In the former case a population of low metallicity stars would be present, either still clustered in e.g., dSph's, or dispersed through the whole cluster potential well. However, a dispersed population can also arise from tidal stripping of galaxies in the course of the cluster evolution (cluster harrassment, cf. Moore et al. 1996), and it will be very hard to distinguish observationally between the two options. Upper limits to a diffused stellar population in clusters have been reported to be at the level of $\sim 20 \%$ of the total cluster light (Melnick, White, \& Hoessel 1977). A promising way to better determine this fraction may come from detecting and counting isolated planetary nebulae that are now being found in clusters (Theuns \& Warren 1997; Arnaboldi et al. 1996). Theuns \& Warren argue that perhaps as much as $40 \%$ of the cluster stellar light comes from a dispersed population, but also point out that this estimate is based on an uncertain frequency of planetary nebulae per unit light of the parent population. This is known to vary by a very large amount among spheroidals, with most metal rich ones having a several times smaller PN productivity compared to metal poor ones (Hui et al. 1993; Ferguson \& Davidsen 1993).

Another possibility is offered by an early generation of massive stars in a now extinct (i.e., $L_{\mathrm{B}} \simeq 0$ ) population produced in a bimodal star formation scenario (Arnaud et al. 1992; Elbaz, Arnaud, \& Vangioni-Flam 1995). One postulates that only massive stars (e.g., 
$M \gtrsim 2 M_{\odot}$ ) would form in starbursts, while the low mass stars that shine today in galaxy clusters would come only later in a quiescent star formation mode. For this model to work fine tuning is required to explain the constancy of the $\mathrm{Fe} M / L$, with the ratio of the star yields of the two star formation modes being the same in all clusters. Moreover, there is little evidence for bimodal star formation in nature. For example, a typical globular cluster formed $\sim 10^{6} M_{\odot}$ of stars in probably less than $10^{6} \mathrm{yr}$, with a star formation rate of $\sim 1 M_{\odot} \mathrm{yr}^{-1}$ per cubic parsec (!), a strong starburst indeed, and yet they were able to produce the wealth of low mass stars without which we would not see them today. Moreover, abundant low mass stars have been detected in Galactic regions with active high mass star formation (Zinnecker, McCaughrean, \& Wilking 1993), and therefore the occurrence of bimodal star formation appears rather implausible. All in all, it appears natural to adopt the view that the metals we see in clusters were produced by the same stellar population which low mass component allows us to see cluster galaxies today.

\subsection{Galaxy Groups}

An inspection to Figures 1-6 immediately reveals that what holds for clusters, either hotter than $\sim 2 \mathrm{keV}$ or brighter than $\sim 4 \times 10^{11} L_{\odot}$, apparently does not hold for

cooler/fainter clusters and groups. I first assume $Z_{\mathrm{ICM}}^{\mathrm{Fe}}$ and $M_{\mathrm{ICM}} / L_{\mathrm{B}}$ ratios for these latter objects at face value, and follow their astrophysical implications. Some of such implications were already mentioned in RCDP, even if at that time data were available for only one object, namely the NGC 2300 group (Mulchaey et al. 1992). The very low values of the $\mathrm{Fe} M / L$ shown in Figure 2 imply that a great deal of iron should have been lost by the groups, hence a major fraction of their baryons along with it. This appears to be in agreement with the ICM mass to light ratio being indeed much lower than in clusters (Figure 4). However, this cannot be the whole story, because besides a lower $\mathrm{Fe} M / L$ several 
groups appear to have a much lower iron abundances compared to clusters (Figure 6). It seems as if, after having shed a major fraction of their original baryon content, such groups had to re-accrete a modest amount of baryons from their surrondings, where pristine gas largely diluted the metal contaminated ejecta of the groups themselves (RCDP). This alternate flow of baryons, out and in groups, may arise as a result of a declining galactic wind activity inside groups, and at least at early times such activity could well have been strong enough to drive gas out of groups (Renzini 1994). However, the whole scenario appears rather contrived, as it may be difficult for groups to reaccrete gas once its adiabat has been raised by energy input from the galactic winds (Kaiser 1991).

There is however another interpretation still open. It is apparent from Figures 2, 4 and 6 that for $k T \gtrsim 2 \mathrm{keV}$ all plotted cluster properties are nearly constant and exhibit very small scatter. Cooler clusters and groups, especially for $k T \lesssim 1 \mathrm{keV}$, show instead a wild range of abundances, gas mass to light ratios, and therefore $\mathrm{Fe} M / L$ values. It is worth emphasizing that the diagnostic tools to get the iron abundance are radically different in the hot clusters compared to the cooler ones. Above $k T \simeq 2 \mathrm{keV}$ iron abundances are derived primarily from the iron-K complex at $\sim 7 \mathrm{keV}$, where lines arise from transitions down to the $\mathrm{K}$ shell of He-like and H-like iron ions. The atomic physics used in this plasma emission models is therefore rather simple. At cooler temperatures instead, iron abundances are derived from the iron-L complex at $\sim 1 \mathrm{keV}$, where lines arise from transitions down to the L shell of progressively more and more complex iron ions as temperature becomes lower and lower. The more complex the ions, the less secure the involved atomic physics calculations, especially the collisional excitation probabilities, and it appears legitimate to entertain the suspicion that much of the observed trends below $\sim 2 \mathrm{keV}$ may just be an artifact of some systematic error in the iron-L diagnostics (cf. AMIOR for an extensive discussion). In the case of the Virgo cluster $(k T \simeq 2.9 \mathrm{keV})$ it has been shown that the iron abundance derived from iron-L lines is in very good agreement with that derived from iron-K lines (AMIOR). 
Hwang et al. (1997) come to the same conclusion analysing a sample of clusters with $k T$ in the $2-4 \mathrm{keV}$ temperature range. Unfortunately, by no means the reliability of the iron-L diagnostics at $k T \simeq 3 \mathrm{keV}$ can prove its reliability at lower temperatures, since then other, much more complex iron ions are involved (AMIOR). In conclusion, the drop of iron abundance below $\sim 1 \mathrm{keV}$ may not be real at all, and therefore it is premature to conclude that groups had to re-accrete baryons at late epochs. It appears that the best way to assess the reliability of iron-L diagnostics at low temperatures will be offered by collecting ASCA observations of objects for which the iron abundance is independently known, such as stars, supernova remnants, starburst galaxies etc. (cf. AMIOR for a preliminary discussion).

While the iron abundances may be in error at low $k T$ values, it is worth emphasizing that the low $M_{\mathrm{ICM}} / L_{\mathrm{B}}$ ratios shown in Figure 4 should be only marginally affected by possible systematic errors in the iron-L diagnostics, i.e., while abundances and $\mathrm{Fe} M / L$ values may be severely underestimated below $\sim 1 \mathrm{keV}$, the low values of the ICM mass to light ratios in groups are more robust, and one can safely conclude that groups are genuinely gas poor, likely to have lost a major part of their original share of baryons. Alternatively, in groups baryon conversion to stars would have been much more efficient than in clusters, proceeding almost to the last "drop" of them, which seems a less plausible interpretation.

\subsection{Clusters vs. Groups and the Formation Epoch of Clusters}

The systematic difference in the $M_{\mathrm{ICM}} / L_{\mathrm{B}}$ ratio of groups relative to rich clusters can

have far reaching consequences. Indeed, for merging groups the $M_{\mathrm{ICM}} / L_{\mathrm{B}}$ ratio should stay the same or decrease slightly (Evrard 1997), therefore failing to meet the high $M_{\mathrm{ICM}} / L_{\mathrm{B}}$ values typical of clusters, which are up to $\sim 30$ times higher than those in groups. This conclusion would be further reinforced were the iron-L diagnostics correct, since merged groups would also have much lower Fe $M / L$ compared to clusters. 
The inference is that rich clusters cannot have formed by merging groups similar to those in the nearby universe. If clusters formed by hierarchical merging, merging should have taken place when groups were still gas rich, likely at high redshift. This favors low- $\Omega$ universes, where little merging and cluster growth takes place at late epochs (low $z$ ), while most of merging activity "switches off" at $1+z \simeq \Omega_{\circ}^{-1} \simeq 5$ in an open universe, or at $1+z \simeq \Omega_{\circ}^{-1 / 3} \simeq 1.5$ in a $\Lambda \mathrm{CDM}$ universe (White 1997). Late accretion of groups onto rich clusters may still take place, but should occur at such a low rate to leave substantially unaffected the ICM mass to light ratio of the clusters. Independent evidence for very little or no cluster evolution all the way out to $z \simeq 0.6$ is now emerging both from optical (Carlberg et al. 1996) as well as X-ray selected cluster samples (Rosati et al. 1997).

This conclusion is further reinforced by some of the considerations in Section 3.1. Indeed, with most of stars in clusters having formed at high redshift $(z \gtrsim 3)$, and with the concomitant production and ejection of iron, much of the heating of the ICM also took place at such early epoch (e.g., Renzini 1994). If clusters were not already in place such heating would have drasticaly affected the baryon content of clusters, as it appears indeed to have done for groups. While small cluster to cluster variations in the baryonic fraction might have been detected (Loewenstein \& Mushotzky 1996b), such variations are tiny compared to the range covered by the $M_{\mathrm{ICM}} / L_{\mathrm{B}}$ ratio of groups in Figures 3 and 4 . Rich clusters appear to be "older" than the bulk of the old stellar population by which they are dominated.

However, one may also envisage a scenario in which groups destined to assemble forming a rich cluster expelled gas at an early stage, the gas remained confined as an intragroup medium, and finally was collected inside the cluster along with the groups. 


\section{The Elemental Ratios in the Intracluster Medium}

It is now well established that in the metal poor stars of the Galactic halo the $\alpha$-elements are enhanced with respect to iron relative to the solar proportions (e.g., Wheeler, Sneden, \& Truran 1989; Bessell, Sutherland, \& Ruan 1991), and a similar enhancement is observed in the metal rich stars of the Galactic bulge (McWilliam \& Rich 1994). The current interpretation of this $\alpha$-element overabundance in the whole Galactic

spheroid appeals to the prompt release of $\alpha$-elements by short living massive stars producing Type II SNs, coupled to the somewhat delayed release of a major fraction of the whole iron by Type Ia SNs (e.g., Greggio \& Renzini 1983; Matteucci \& Greggio 1986; Ruiz-Lapuente, Burkert, \& Canal 1996). Various degrees of $\alpha$-element enhancement can thus be obtained, depending on the adopted time scale of the iron release by SNIa's relative to the time scale of star formation. This scenario is referred to as the "standard chemical model" for the Galactic chemical evolution (RCDP). Moreover, population synthesis methods indicate that an $\alpha$-element enhancement may also be present in ellipticals, reaching up to $0.2-0.3$ dex in the most massive ones (e.g., Worthey, Faber, \& Gonzales 1992; Davies, Sadler, \& Peletier 1993). By analogy with the Galactic spheroid, the favored interpretation has been in terms of the relative roles of the two SN types during the fast formation process of ellipticals.

A fast completion of star formation (on time scales of, say $\lesssim$ few $10^{8} \mathrm{yr}$ ) implies that a fraction of the iron released by SNIa's should flow directly out of galaxies during an early galactic wind phase, without ever being incorporated into stars. If so, a chemical asymmetry should be established between galaxies and the ICM, with galaxies being slightly overabundant in $\alpha$-elements relative to iron, and the ICM being slightly overabundant in iron relative to the $\alpha$-elements (RCDP). The size of this asymmetry is hard to predict theoretically, as it depends on the relative time scales of star formation on the one hand, and of the SNIa iron release on the other, with both time scales being poorly known. The 
effect is not expected to be large. If all the iron from SNIa were ejected from galaxies the $\alpha$-deficiency in the ICM would be $[\alpha / \mathrm{Fe}] \sim-0.4$ (RCDP). The actual deficiency may be much smaller than this, since part of the SNIa iron is likely to be incorporated into stars, while SNII products are also ejected from galaxies.

Early attempts at measuring the abundance of $\alpha$-elements in the ICM indicated a possible overabundance of oxygen relative to iron (Canizares et al. 1982) and a near solar Si/Fe ratio (Mushotzky et al. 1981), but both with large statistical and systematic uncertainty. $A S C A$ has now provided much better data, and Mushotzky (1994) has initially reported a fairly high $\alpha$-element enhancement, with $<[\alpha / \mathrm{Fe}]>\simeq+0.4$ (from his Table 3). More recently Mushotzky et al. (1996) have revised down this estimate, and report detailed elemental abundances for several $k T=3-4 \mathrm{keV}$ clusters, all showing a moderate $\alpha$-element enhancement. Uncertainties in the abundances are reported to be of about a factor of two, at the $90 \%$ confidence level. Taking a global average for $\mathrm{O}, \mathrm{Ne}, \mathrm{Mg}, \mathrm{Si}$, and $\mathrm{Fe}$, one obtains a modest $\langle[\alpha / \mathrm{Fe}]>\simeq+0.2$. This is still in conflit with the predicted asymmetry, and argues for not only the galaxies, but the ICM as well being dominated by SNII products (Loewenstein \& Mushotzky 1996a). If so, this would demonstrate the impossibility to extend to galaxy clusters the standard chemical model that apparently holds for the Galactic nucleosynthesis (AMIOR).

However, Ishimaru \& Arimoto (1997) have recently pointed out that the small $\alpha$-element enhancement in the ICM comes from Mushotzky et al. (1996) having assumed reference solar abundances from "photospheric" model atmosphere analysis. The result is different if one uses "meteoritic" abundances instead, because the meteoritic iron abundance is $\sim 0.16$ dex lower than the photospheric value (Anders \& Grevesse 1989). After noting that the meteoritic iron is now more generally adopted, Ishimaru \& Arimoto conclude that the $\alpha$-element enhancement in the ICM virtually disappears, going down from $\sim 0.2$ to only 
$\sim 0.04$ (consistent with no enhancement or even small depletion, given the reported errors)

At this stage it is safe to conclude that there is no strong evidence for an enhanced $\alpha$-element proportion in the ICM, and that the galaxy-ICM chemical asymmetry - if it exists - must be small and probably hard to detect given the current errors in abundance determinations. Abundance ratios in the cluster as a whole (including stars and ICM together) are therefore very close to solar, as expected in the frame of the standard chemical model (RCDP). Therefore, the applicability of the standard chemical model to clusters is not invalidated, and the possibility of a major contribution from SNIa's to the iron enrichment of clusters remains viable.

\section{The Metallicity of the Present Day Universe and the Past History of Star Formation}

Clusters of galaxies are the largest entities for which we can measure the metallicity. It is important to notice that the mass of iron in the ICM is comparable to that locked into galaxies, having assumed the average abundance of stars in galaxies to be solar (RCDP). While the abundances in either the ICM or galaxies are independent of the distance scale, the cluster average abundance does actually depend on the assumed Hubble constant, because so do both the mass in galaxies and $M_{\mathrm{ICM}}$, and each of them in a different way. I assume as prototypical the Coma cluster values adopted by White et al. (1993): $M_{\mathrm{ICM}} \simeq 5.5 \times 10^{13} h^{-5 / 2} M_{\odot}$ and $M_{*} \simeq 10^{13} h^{-1} M_{\odot}$. and derive for the cluster iron abundance:

$$
Z_{\mathrm{CL}}^{\mathrm{Fe}}=\frac{Z_{\mathrm{ICM}}^{\mathrm{Fe}} M_{\mathrm{ICM}}+Z_{*}^{\mathrm{Fe}} M_{*}}{M_{\mathrm{ICM}}+M_{*}}=\frac{5.5 Z_{\mathrm{ICM}}^{\mathrm{Fe}} h^{-5 / 2}+Z_{*}^{\mathrm{Fe}} h^{-1}}{5.5 h^{-5 / 2}+h^{-1}}
$$

where $Z_{*}^{\mathrm{Fe}}$ is the average abundance of stars in galaxies and $M_{*}$ is the mass in stars.

With $Z_{\mathrm{ICM}}^{\mathrm{Fe}}=0.3$ solar and $Z_{*}^{\mathrm{Fe}}=1$ solar, equation (1) gives a global cluster abundance of $0.34,0.37$, and 0.41 times solar, respectively for $h=0.5,0.75$, and 1 . Under the same 
assumptions, the ratio of the iron mass in the ICM to the iron mass locked into stars is:

$$
\frac{Z_{\mathrm{ICM}}^{\mathrm{Fe}} M_{\mathrm{ICM}}}{Z_{*}^{\mathrm{Fe}} M_{*}} \simeq 1.65 h^{-3 / 2},
$$

or 4.6, 2.5, and 1.65, respectively for $h=0.5,0.75$, and 1 . Note that with the adopted values for the quantities in equation (2) most of the iron is in the ICM, rather than now locked into stars, especially for low values of $H_{\circ}$. These estimates could be somewhat decreased if clusters contain a sizable population of stars not bound to censed individual galaxies (cf. Section 3.1). The iron content of galaxies may also have been underestimated, because so does a luminosity-weighted abundance compared to the mass-weighted abundance (e.g., Greggio 1997). In addition, the galaxy (baryonic) $M_{*} / L$ ratio might be higher than estimated by White et al. (1993), i.e., $\left\langle M_{*} / L_{\mathrm{B}}\right\rangle=6.4 h$. All these effects together may contribute to reduce the ICM to galaxies iron ratio below the somewhat embarrasingly large values given above.

With the adopted masses and iron abundances for the two baryonic components one can also evaluate the total cluster Fe $M / L$. One needs to specify the average $M_{*} / L_{\mathrm{B}}$ ratio, and adopting for consistency the value given by White et al. (1993), one gets:

$$
\frac{M_{\mathrm{Fe}}^{\mathrm{ICM}}+M_{\mathrm{Fe}}^{*}}{L_{\mathrm{B}}} \simeq 1.3 \times 10^{-2}\left(1.65 h^{-1 / 2}+h\right) \quad\left(M_{\odot} / L_{\odot}\right),
$$

or $\mathrm{Fe} M / L=0.037$ or $0.034 M_{\odot} / L_{\odot}$, respectively for $h=0.5$ and 1 . The total $\mathrm{Fe} M / L$ is therefore fairly insensitive to the adopted distance scale, and is close to the value previously estimated (i.e., $0.03 M_{\odot} / L_{\odot}, \mathrm{RCDP}$; Renzini 1994). Simple calculations (cf. RCDP) show that to reproduce this value one needs either a fairly flat IMF $(x \simeq 0.9)$ if all iron is attributed to SNII's, or a major contribution from SNIa's, if one adopts a Salpeter IMF $(x=1.35)$. The former option dictates a substantial $\alpha$-element enhancement, similar to the values observed in the Galactic halo $([\alpha / \mathrm{Fe}] \simeq+0.5)$. The latter option instead predicts near solar proportions for the cluster as a whole. On the basis of the discussion in Section 
4 one concludes in favor of the second option. Moreover, the total metal mass to light ratio of clusters is $\sim 0.3 M_{\odot} / L_{\odot}$, given that in the solar proportion iron accounts for about $10 \%$ of all metals. There is no doubt that the bulk of such metals other than iron - hence of the metals as whole - was produced by SNIIs. Now, the number of SNIIs exploded at early times in a stellar population that has faded to luminosity $L_{\mathrm{B}}$ when aged to $\sim 15$ Gyr is $\sim 0.1 \times L_{\mathrm{B}} / L_{\odot}(\mathrm{RCDP})$. Therefore, to produce the observed metal mass to light ratio of clusters each SNII must have contributed on average $\sim 3 M_{\odot}$ of heavy elements, which is in agreement with current nucleosynthesis calculations (e.g., Woosley \& Weaver 1995).

A critical issue is to what extent the cluster global metallicity, and the ICM to galaxies iron share are representative of the low $-z$ universe as a whole. For example, Madau et al. (1996) adopt $H_{\circ}=50$, a stellar mass density parameter $\Omega_{*}=0.0036$, a baryon mass density parameter $\Omega_{\mathrm{b}}=0.05$, an average solar metallicity for the stars, and a negligible metal content for the intergalactic medium (IGM), that comprises the vast majority of the baryons. With these assumptions the metallicity of the present day universe is $\sim 1 \times 0.0036 / 0.05=0.07$ solar, or $\sim 5$ times lower than the measured value in clusters of galaxies. In the same frame, the fraction of baryons in galaxies (stars) is also $\sim 7 \%$ globally, which compares to $\sim 1 /\left(1+5.5 h^{-3 / 2}\right)$ in clusters, or $\sim 6 \%$ and $\sim 10 \%$, respectively for $h=0.5$ and 0.75 . Therefore, it appears that the efficiency of baryon conversion into galaxies and stars $\left(\Omega_{*} / \Omega_{\mathrm{b}}\right)$ adopted by Madau et al. (1996) is nearly the same as that observed in clusters, which supports the notion of clusters being representative of the low-z universe. The metallicity of the clusters is however $\sim 5$ times higher than the metallicity of the low- $z$ universe as conservatively adopted by Madau et al.. The difference comes from having attributed to the IGM a very low metallicity, hence assuming field galaxies losing only a negligible amount of metals. This implies a factor $\sim 5$ lower efficiency in metal production per unit mass turned into stars. I will discuss the two aspects in turn. 
Is there any reason why most of the produced metals should be ejected by cluster galaxies, and instead fully retained by field galaxies, while reaching the same average stellar metallicity? The only hint for a systematic cluster/field difference may be offered by ram pressure stripping, that to some extent should be active in clusters but not in the field. However, in clusters iron was most likely ejected from galaxies as a result of supernova heating, rather than stripped by ram pressure (cf. RCDP). In particular, ram pressure stripping cannot have played a major role because as shown by Figure 2 the $\mathrm{Fe} M / L$ is the same in clusters with moderate velocity dispersion (or equivalently, $k T \simeq 2 \mathrm{keV}$ ) as in clusters with high velocity dispersion ( or $k T \simeq 10 \mathrm{keV}$ ). Were ram pressure important one would have expected the Fe $M / L$ to increase with ICM temperature. There appears also to be no strong argument for field galaxies having shed much less iron than cluster galaxies. The small starburst galaxies making the excess blue galaxy counts (Lilly et al. 1995) are indeed likely to have ejected a sizable amount of metals before "fading to oblivion".

Seemingly, there are no strong arguments supporting the factor of $\sim 5$ difference in metal production efficiency, unless one is willing to postulate a flatter stellar IMF in cluster relative to field galaxies. The difference might be somewhat smaller if one allows for the possibility that there are more stars in clusters than adopted here (cf. Section 3.1). However, if such stars escaped detection within well studied clusters, they may have gone unnoticed in the field as well. Therefore, it would appear quite ad hoc to appeal to a much higher metal productivity of stellar populations in galaxy clusters compared to their field counterpart, and it appears reasonable to conclude that the global metallicity of the present day universe may well be nearly the same as that observed in galaxy clusters, i.e., $\sim 0.3-0.4$ times solar. If so, there should be a comparable share of metals in the field IGM, as there is in the cluster ICM.

While it appears that the global, time-averaged rate of metal production in the universe 
may have been underestimated by perhaps as much as a factor $\sim 5$, this does not necessarily imply that the global, time-averaged rate of star formation was also underestimated by the same factor. More simply, the metal productivity of galaxies per unit of their present mass may have been underestimated by Madau et al., if the cluster galaxies productivity is to be taken as representative of the universe as a whole. One cannot exclude that actual average rate of star formation may have been underestimated, which would be the case if a population exists of unaccounted stars in clusters and in the field.

\section{Conclusions}

In this paper several relations coming from combining galaxy cluster X-Ray and optical data have been presented and discussed, and astrophysical inferences have been drawn from them. The main results can be summarized as follows.

1. The ICM iron mass to optical light ratio of clusters with $k T \gtrsim 2 \mathrm{keV}$ appears to be constant, with a small dispersion which is fully consistent with observational errors. Among these clusters also the iron abundance and the ICM mass to light ratio appear to be constant with small dispersion.

2. Among poor clusters and groups, instead, the Fe $M / L$, the ICM mass to light ratio, and the iron abundance all drop by orders of magnitude compared to the corresponding values typical of the rich clusters. This is especially evident for ICM temperatures below $\sim 1 \mathrm{keV}$.

3. The constancy of the Fe $M / L$ among clusters indicates that clusters did not lose an appreciable fraction of their original share of the cosmic baryons. It also argues for the stellar initial mass function being nearly the same in all clusters, as well as for the average stellar ages being also the same from cluster to cluster, within less than a factor $\sim 2$.

4. As a major fraction of the cluster optical luminosity comes from ellipticals and bulges, 
the stellar population responsible for the production of the iron and the other heavy elements now in the ICM is most naturally identified with the high mass tail of the same population now surviving in ellipticals and bulges. Since such spheroid populations formed at high redshift $(z \gtrsim 3)$, much of the iron should have been ejected from galaxies at a very early stage. This is in agreement with the observed iron abundance in moderate redshift clusters being the same as in local clusters.

5. The possibility remains that a non-negligible fraction of the cluster stellar population resides out of censed galaxies. If so, a corresponding fraction of the ICM metals could have been manufacted by this dispersed stellar population. In principle, part of the metals could have been produced by a now extinct stellar population, generated in a bimodal star formation mode. It is argued that this hypothesis is rather implausible.

6. The drop of the ICM mass to light ratio in groups indicates that - contrary to clusters they have experienced a major loss of gas (baryons) in the course of their evolution. The very low metallicity indicated by current X-ray diagnostics for most of these groups, if real, suggests that baryons should have been re-accreted after an initial de-gassing, which seems rather contrived. However, it is possible that current iron- $\mathrm{L}$ diagnostics for $k T \lesssim 1 \mathrm{keV}$ is affected by systematic errors.

7. The large differences between the cluster and the group ICM mass to light ratios suggest that clusters did not form by agglomerating groups similar to the present day ones. If such agglomeration took place, it must have occurred while the groups were still gas rich, i.e., before major star formation took place thus propelling part of the baryons out of them. This argues for the assembly of rich clusters having been completed at high redshift, which favors low- $\Omega$ universes.

8. There is not much evidence for a predicted chemical asymmetry in the $\alpha$-element to iron ratios between the ICM and the stellar component of clusters. However, data are consistent 
with no $\alpha$-element enhancement in the ICM, and therefore with a combined SNIa and SNII enrichment to give near solar elemental proportions on the whole cluster scale, as predicted by the standard model for the Galactic chemical evolution. In particular, there appears to be no need to invoke a special IMF or a suppression of SNIa's in clusters, compared to our own Galaxy.

9. It is emphasized that the overall cluster metallicity is about 5 times higher than the currently adopted average metallicity of the present-day universe, in spite of a similar fraction of the baryons having been converted into galaxies and stars in the clusters as well as in the general field. Possible origins of the difference (or discrepancy) are discussed, including an underestimate of the efficiency of metal production per unit mass of baryons converted into stars.

10. The amount of iron (metals) in an undetected, probably hot intergalactic medium comprising most of the bayons is predicted to be comparable to - or even larger than - the amount of iron now locked into stars in the present day universe. The metal abundance of this IGM is predicted to be $\sim 1 / 3$ solar, if the metal productivity of stellar populations is the same in the field as it is in clusters.

I am grateful to Mauro Giavalisco and Piero Madau for their support and for useful discussions about the metallicity of the present day universe and the integrated past star formation activity, and to Ralph Bender and an anonymous referee for constructive comments. I wish to thank Richard Ellis and Trevor Ponman for stimulating discussions, and for their invitation to participate at the Royal Astronomical Society meeting of October 11, 1996, and the Pontificial Academy of Sciences for its invitation to attend the Vatican Conference on "The Emergence of Structure in the Universe at the Level of Galaxies", November 25-29, 1996, as at these meetings I had the opportunity to report and discuss in a friendly environment the results now presented in this paper. 


\section{REFERENCES}

Anders, E., \& Grevesse, N. 1989, Geochimica et Cosmochimica Acta, 53, 197

Aragon-Salamanca, A., Ellis, R.S., Couch, W.J. \& Carter, D. 1993, MNRAS, 262, 764

Arimoto, N., \& Yoshii, Y. 1987, A\&A, 173, 23

Arimoto, N., Matsushita, K., Ishimaru, Y., Ohashi, T., \& Renzini, A. 1997, ApJ, 477, 128 (AMIOR)

Arnaboldi, M., Freeman, K.C., Mendez, R.H., Capaccioli, M., Ciardullo, R., Ford, H., Gerhard, O., Hui, X., Jacoby, G.H., Kudritzki, R., \& Quinn, P.J. 1996, ApJ, 472, 145

Arnaud, M., Rothenflug, R., Boulade,O., Vigroux, R., \& Vangioni-Flam, E. 1992, A\&A, 254,49

Bender, R., Ziegler, B., \& Bruzual, G.A. 1996, ApJ, 436, L51

Bessell, M.S., Sutherland, R.S., \& Ruan, K. 1991, ApJ 383, L71

Bower, R.G., Lucey, J.R. \& Ellis, R.S. 1992, MNRAS, 254, 613

Canizares, C.R., Clark, G.W., Jernigan, J.G., \& Markert, T.H. 1982, ApJ, 262, 33

Carlberg, R.G., Morris, S.L., Yee, H.K.C., Ellingson, E. 1996, astro-ph 9612169

Ciotti, L., D’Ercole, A., Pellegrini, S., \& Renzini, A. 1991, ApJ, 376, 380

David, L.P., Arnaud, K.A., Forman, W., \& Jones, C. 1990, ApJ, 356, 32

David, L.P., Jones, C., Forman, W., \& Daines, S. 1994, ApJ, 428, 544

Davies, R.L., Sadler, E.M., Peletier, R.F., 1993, MNRAS, 262, 650 
Davis, D.S., \& White, R.E. III, 1996, ApJ, 470, L35

Dickinson, M. 1995, in Fresh Views of Elliptical Galaxies, ed. A. Buzzoni, A. Renzini, \& A. Serrano, ASP Conf. Ser. 86, 283

Elbaz, D., Arnaud, M., \& Vangioni-Flam, E. 1995, A\&A, 303, 345

Ellis, R.S., Smail, I., Dressler, A., Couch, W.J., Oemler, A. Jr., Butcher, H., \& Sharples, R.M. 1996, astro-ph 9607154

Evrard, A.E. 1997, MNRAS, in press (astro-ph 9701148)

Ferguson, H.C., \& Davidsen, A.F. 1993, ApJ, 408, 92

Greggio, L. 1997, MNRAS, 285, 151

Greggio, L., \& Renzini, A. 1983, Mem. SAIt, 54, 311

Hui, X., Ford, H.C., Ciardullo, R., \& Jacobi, G.H. 1993, ApJS, 88, 423

Hwang, U., Mushotzky, R.F., Loewenstein, M., Markert, T.H. Fukazawa, Y., \& Matsumoto, H. 1997, ApJ, 476, 560

Ishimaru, Y., \& Arimoto, N. 1997, PASJ, in press (astro-ph 9702036)

Jablonka, P., \& Alloin, D. 1995, A\&A, 298, 361

Kaiser, N. 1991, ApJ, 383, 104

Kodama, T., \& Arimoto, N. 1997, A\&A, in press (astro-ph 9609160)

Larson, R.B. 1974, MNRAS, 169, 229

Lilly, S.J., Tresse, L., Hammer, F., Crampton, D., \& Le Fèvre, O. 1995, ApJ, 455, 108

Loewenstein, M., \& Mushotzky, R.F. 1996a, ApJ, 466, 695 
1996b, astro-ph 9608111

Madau, P., Ferguson, H.C., Dickinson, M.E., Giavalisco, M., Steidel, C.C., \& Fruchter, A. 1996, MNRAS, 283, 1388

Matteucci, F. 1992, ApJ, 397, 32

Matteucci, F., \& Greggio, L. 1986, A\&A, 154, 279

Matteucci, F., \& Vettolani, G. 1988, A\&A, 202, 21

McWilliam, A., \& Rich, R.M. 1994, ApJS, 91, 749

Melnick, J., White, S.D.M., \& Hoessel, J. 1977, MNRAS, 180, 207

Mitchell, R., Ives, J., \& Culhane, L. 1975, MNRAS, 175, 29

Moore, B., Katz, N., Lake, G., Dressler, A., \& Oemler, A. 1996, Nature, 379, 613

Mulchaey, J.S., Davis, D.S., Mushotsky, R.F., \& Burstein, D. 1993, ApJ, 404, L9 . 1966, ApJ, 456, 80

Mushotzky, R.F. 1994, in Clusters of Galaxies, ed. F. Durret, A. Mazure, \& J. Tran Thanh Van (Gyf-sur-Yvette: Editions Frontières), p. 167

Mushotzky, R.F., Loewenstein, M., Awaki, H., Makishima, K., Matsushita, K., \& Matsumoto, H., 1994, ApJ, 436, L79

Mushotzky, R.F., Loewenstein, M., Arnaud, K., Tamura, T., Fukazawa, Y., Matsushita, K., Kikuchi, K., \& Hatsukade, I., 1996, ApJ, 466, 686

Nath, B.B., \& Chiba, M. 1995, ApJ, 454, 604 
Ohashi, T., Fukazawa, Y., Ikebe, Y., Ezawa, H., Tamura, T., \& Makishima, K. 1995, in New Horizons of X-ray Astronomy, ed. F. Makino \& T. Ohashi (Tokyo: University Academic Press), p. 234

Ortolani, S., Renzini, A., Gilmozzi, R., Marconi, G., Barbuy, B., Bica, E., \& Rich, R.M., 1995, Nature, 377, 701

Pahre, M.A., Djorgovski, S.G., \& de Carvalho, R.R. 1997, in Galaxy Scaling Relations: Origins, Evolution and Applications, ed. L. da Costa \& A. Renzini (Berlin: Springer), in press

Pei, Y.C., \& Fall, M. S.M. 1995, ApJ, 454, 69

Ponman, T.J., et al. 1994, Nature, 369, 462

Renzini, A. 1994, in Clusters of Galaxies, ed. F. Durret, A. Mazure, \& J. Tran Thanh Van (Gyf-sur-Yvette: Editions Frontières), p. 221

- 1995, in Stellar Populations, ed. P.C. van der Kruit \& G. Gilmore (Dordrecht: Kluwer), p. 325

Renzini, A., \& Ciotti, L. 1993, ApJ, 416, L49

Renzini, A., Ciotti, L., D’Ercole, A., \& Pellegrini, S. 1993, ApJ, 419, 52 (RCDP)

Rosati, P., Della Ceca, R., Giacconi, R., \& Norman, C. 1997, Preprint

Ruiz-Lapuente,P., Burkert, A., \& Canal, R. 1996, ApJ 447, L69

Serlemitsos, P., Smith, B., Boldt, E., Hold, S.S., \& Swank, J. 1976, ApJ, 211, L63

Trentham, N. 1994, Nature, 372, 157

Theuns, T., \& Warren, S.J. 1997, MNRAS, in press 
Tsuru, T. 1993, PhD Thesis, University of Tokyo, ISAS RN 528

van Dokkum, P.G., \& Franx, M. 1996, MNRAS, 281, 985

Vigroux, L. 1977, A\&A, 56, 473

Wheeler, J.C., Sneden, C., \& Truran, J.W.Jr. 1989, ARA\&A, 27, 279

White, S.D.M. 1997, in The Early Universe with the VLT, ed. J. Bergeron (Berlin: Spinger), p. 219

White, S.D.M., Navarro, J.F., Evrard, A.E., \& Frenk, C.S. 1993, Nature, 366, 429

Woosley, S.E., \& Weaver, T.A. 1995, ApLS, 101, 181

Worthey, G., Faber, S.M., \& González, J.J. 1992, ApJ, 398, 69

Worthey, G., Trager, S.C., \& Faber S.M. 1995, in Fresh Views of Elliptical galaxies, ed. A. Buzzoni, A. Renzini, \& A. Serrano, ASP Conf. Ser. 86, 203

Zinnecker, H., McCaughrean, M.J., \& Wilking, B.A. 1993, in Protostars and Planets III, ed. E.H. Levy \& J.I. Lunine (Tucson: Univ. Arizona), p. 429 
Fig. 1. - The iron mass to light ratio of the ICM of clusters and groups as a function of the total optical luminosity $L_{\mathrm{B}}$ of the cluster galaxies. Data are taken from the following sources: filled circles: Arnaud et al. (1992); filled triangles: Tsuru (1993); open triangle: David et al. (1994a); open square: Mulchaey et al. (1993); filled square: Ponman et al. (1994); open circles: Mulchaey et al. (1996).

Fig. 2.- The same as Figure 1 but as a function of the ICM temperature.

Fig. 3.- The ICM mass to light ratio, i.e., the mass of the ICM per unit light of the cluster galaxies, as a function of the total optical luminosity $L_{\mathrm{B}}$ of the cluster galaxies. The same objects as in Figure 1 are displayed.

Fig. 4. - The same as Figure 3 but as a function of the ICM temperature.

Fig. 5.- The iron abundance in the ICM for the objects in Figure 1 as a function of the total optical luminosity $L_{\mathrm{B}}$ of the cluster galaxies.

Fig. 6. - The same as Figure 5 but as a function of the ICM temperature. Data for six clusters at moderately high redshift $(\langle z\rangle \simeq 0.33)$ are also included and represented by small filled circles (Allen et al. 1996; Donahue 1996; Matsuura et al. 1996; Schindler et al. 1997). Note that for $k T>\sim 3 \mathrm{keV}$ the iron abundance is derived from the iron-K complex, while for lower temperatures the iron-L complex is used. 


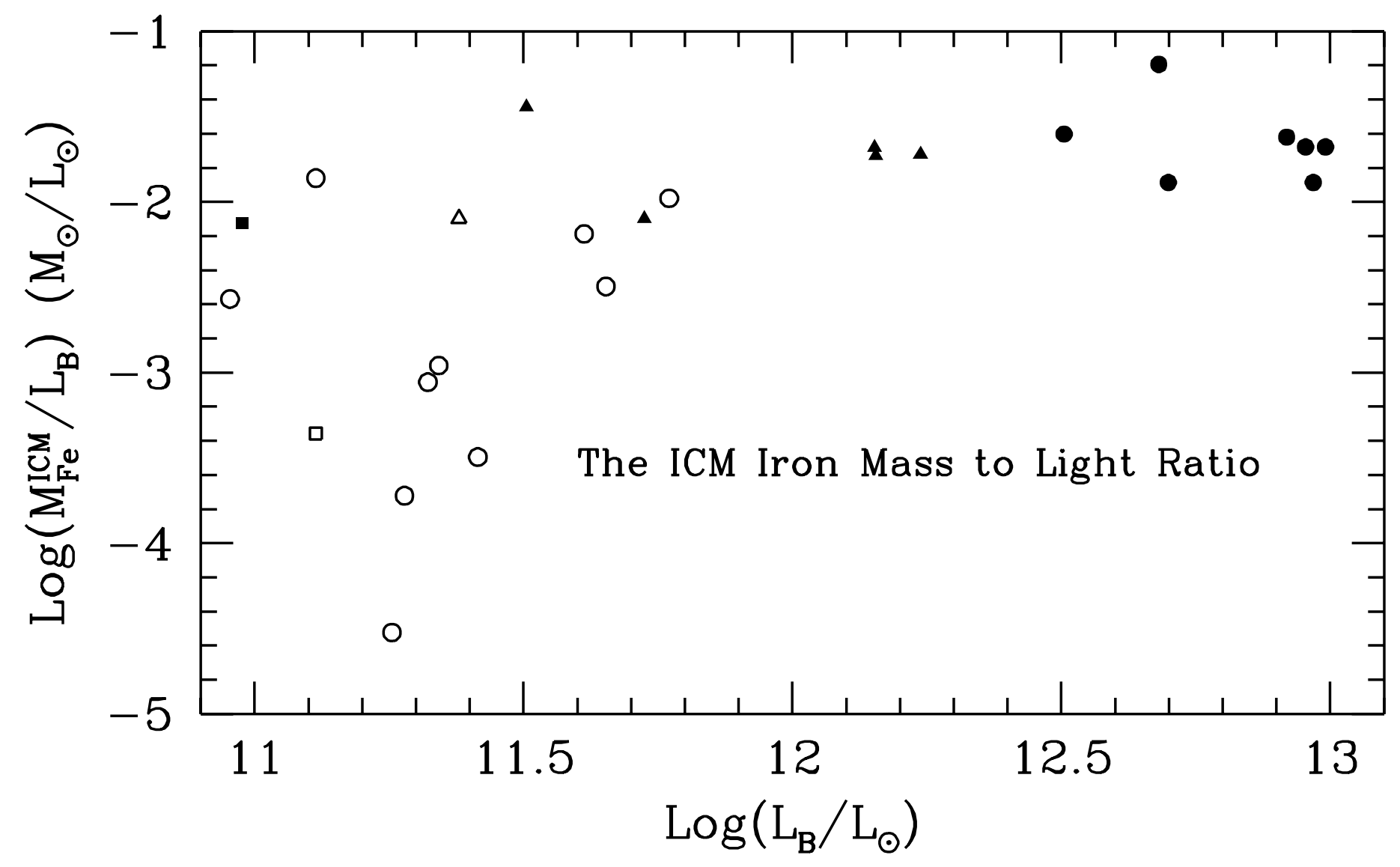




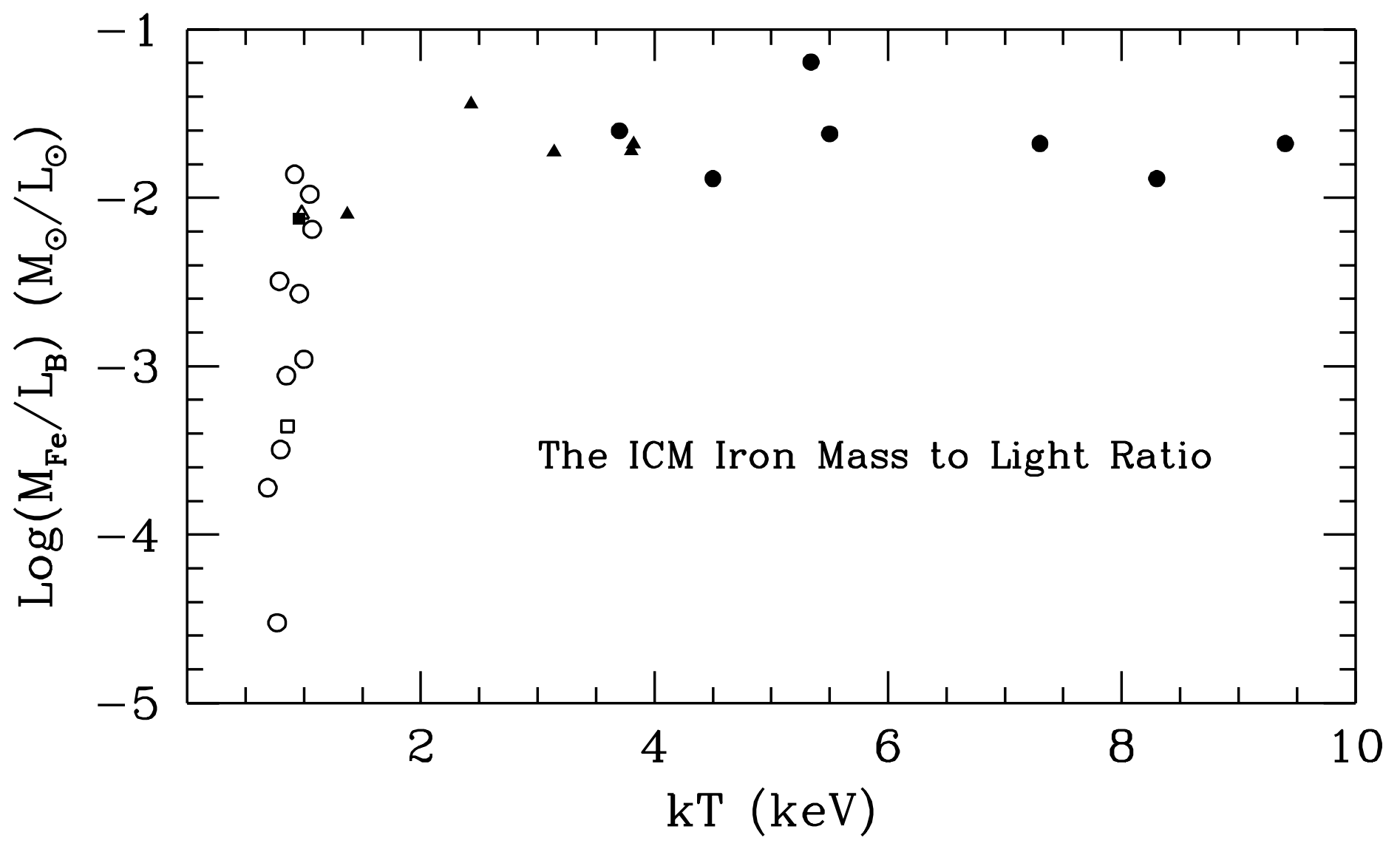




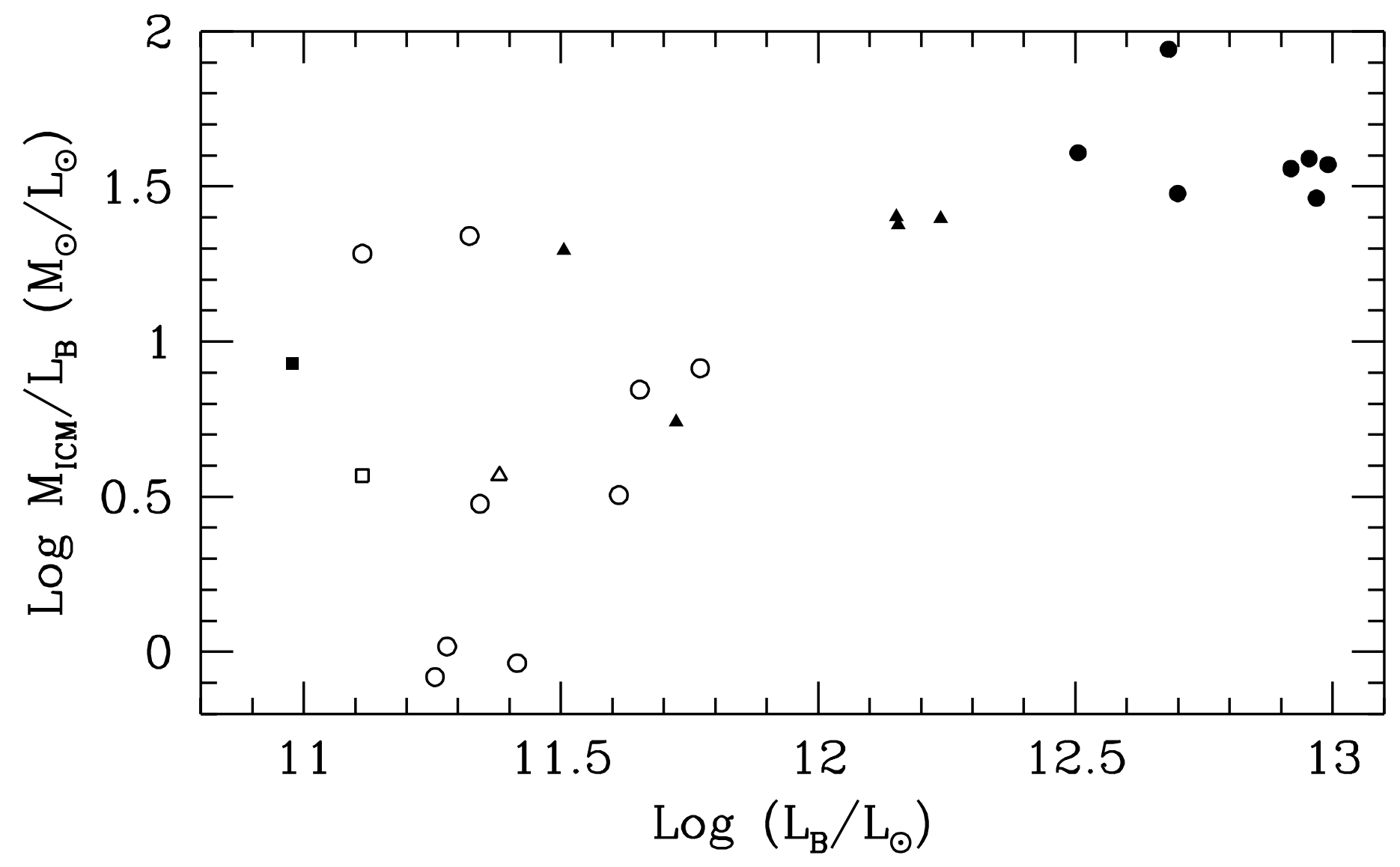




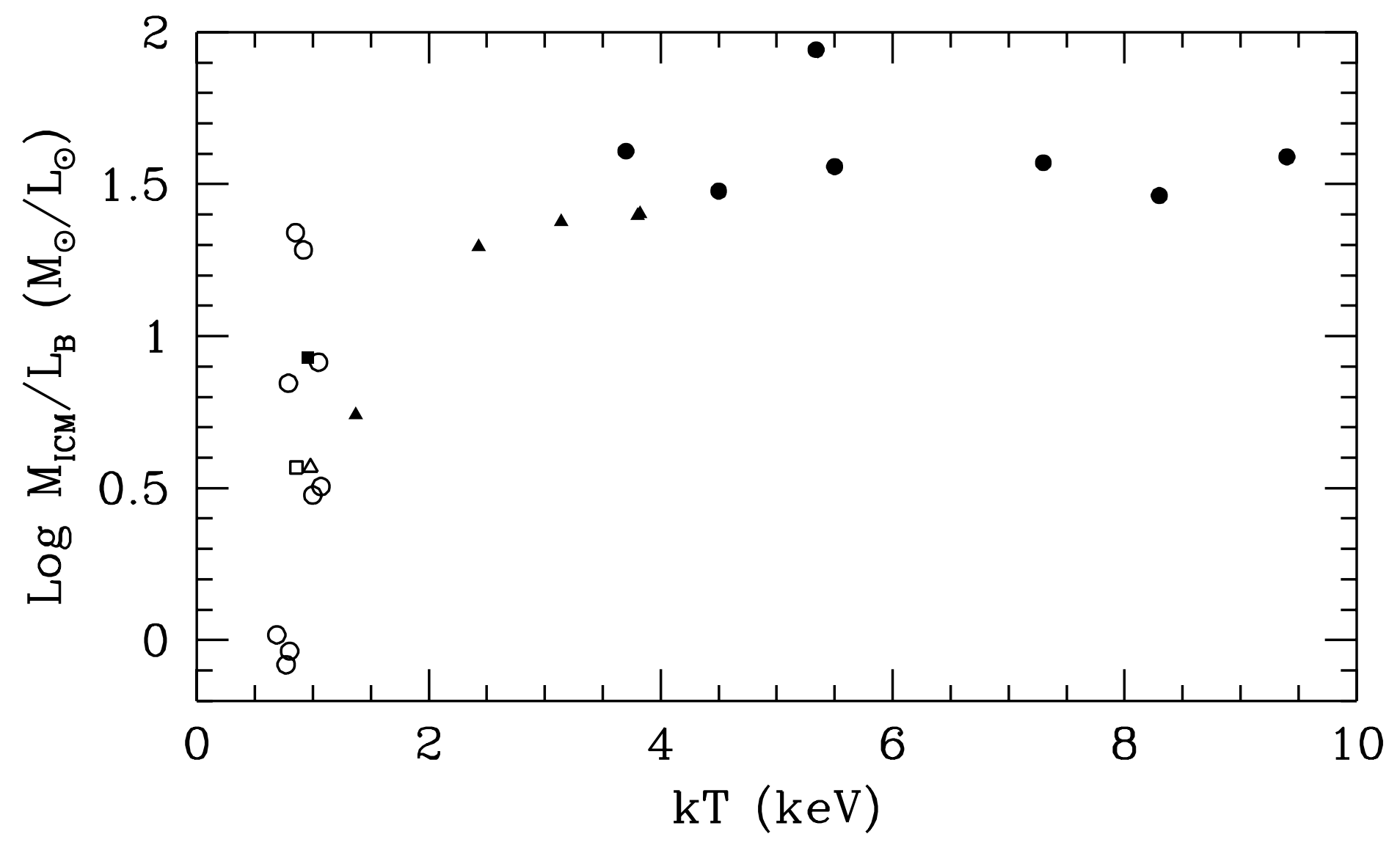




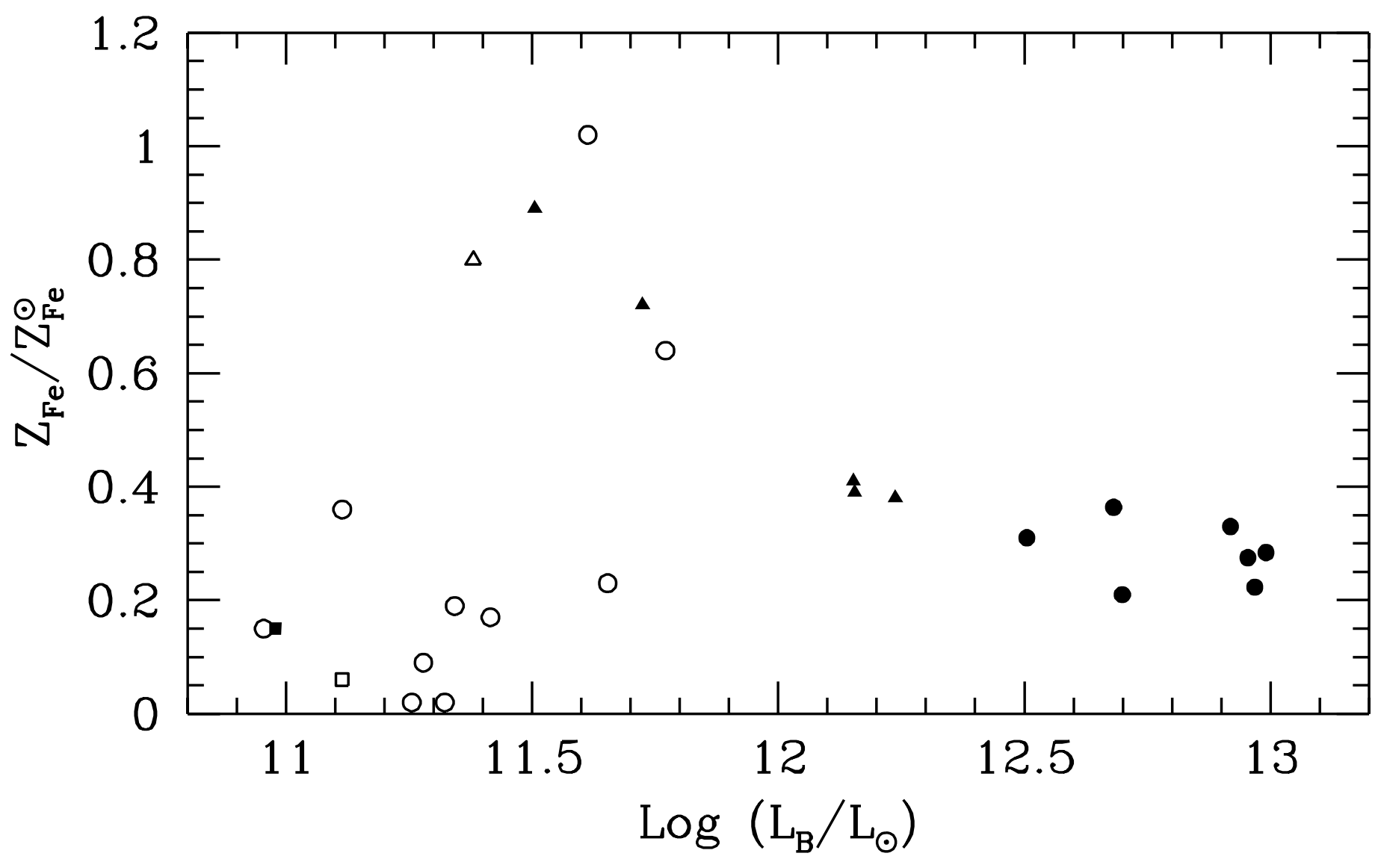




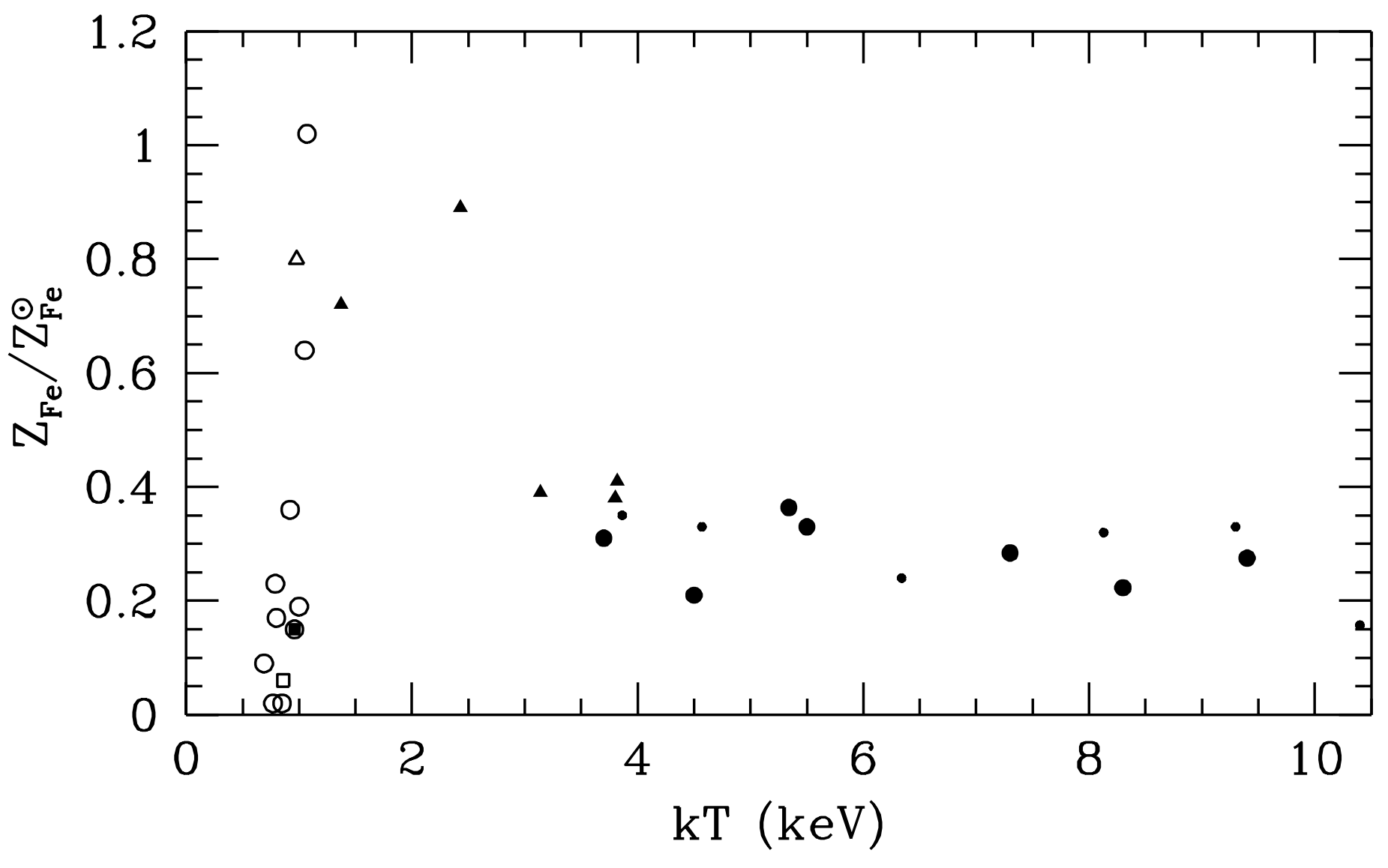

